\title{
Recrystallization of amorphous nanotracks and uniform layers generated by swift-ion-beam irradiation in lithium niobate
}

\author{
M.L. Crespillo • O. Caballero-Calero • V. Joco • \\ A. Rivera · P. Herrero · J. Olivares · F. Agulló-López
}

\begin{abstract}
The thermal annealing of amorphous tracks of nanometer-size diameter generated in lithium niobate $\left(\mathrm{LiNbO}_{3}\right)$ by Bromine ions at $45 \mathrm{MeV}$, i.e., in the electronic stopping regime, has been investigated by $\mathrm{RBS} / \mathrm{C}$ spectrometry in the temperature range from $250^{\circ} \mathrm{C}$ to $350^{\circ} \mathrm{C}$. Relatively low fluences have been used $\left(<10^{12} \mathrm{~cm}^{-2}\right)$ to produce isolated tracks. However, the possible effect of track overlapping has been investigated by varying the fluence between $3 \times 10^{11} \mathrm{~cm}^{-2}$ and $10^{12} \mathrm{~cm}^{-2}$. The annealing process follows a two-step kinetics. In a first stage (I) the track radius decreases linearly with the annealing time. It obeys an Arrhenius-type dependence on annealing temperature with activation energy around $1.5 \mathrm{eV}$. The second stage (II) operates after the track radius has decreased down to around
\end{abstract}

$2.5 \mathrm{~nm}$ and shows a much lower radial velocity. The data for stage I appear consistent with a solid-phase epitaxial process that yields a constant recrystallization rate at the amorphouscrystalline boundary. HRTEM has been used to monitor the existence and the size of the annealed isolated tracks in the second stage. On the other hand, the thermal annealing of homogeneous (buried) amorphous layers has been investigated within the same temperature range, on samples irradiated with Fluorine at $20 \mathrm{MeV}$ and fluences of $\sim 10^{14} \mathrm{~cm}^{-2}$. Optical techniques are very suitable for this case and have been used to monitor the recrystallization of the layers. The annealing process induces a displacement of the crystallineamorphous boundary that is also linear with annealing time, and the recrystallization rates are consistent with those measured for tracks. The comparison of these data with those previously obtained for the heavily damaged (amorphous) layers produced by elastic nuclear collisions is summarily discussed.

\section{Introduction}

It is well known $[1,2]$ that elastic nuclear collisions between an incident ion-beam and a material constitutes a mechanism of energy losses (nuclear stopping power $S_{n}$ ) that is dominant at low energies (roughly, $E<A \mathrm{MeV}$, with $A$ the mass number). When the energy transferred to the target atom overcomes a certain value (displacement energy $E_{D}$ ), the atom is ejected from its lattice site and point defects (Frenkel pairs) are generated $[1,3]$. At sufficiently high fluences (in the range $10^{15}-10^{17} \mathrm{~cm}^{-2}$ ), defect clustering develops, and a quasi-amorphous layer develops monotonically at the end of the ion range, i.e., the region where the ions are implanted. 
On the other hand, abundant experimental evidence is being gathered showing that defects can be also cumulatively created by every individual ion impact in the regime where the electronic stopping power $S_{e}$ is dominant [4]. The structure of these defects is not yet clear, although some detailed data on InP [5] do not show significant differences in the atomic structure of samples amorphized by electronic excitation and elastic collisions. It has been definitely ascertained by several techniques that linear amorphous tracks can be generated along the ion trajectory if the electronic stopping power is above a certain threshold value [6-11]. These tracks have a nanometer diameter and are surrounded by a region of strongly defective material (halo). When the irradiation fluence is high enough so that individual tracks overlap, a rather homogeneous amorphous layer is generated $[12,13]$. For $\mathrm{LiNbO}_{3}$, the final amorphous layer has a well-defined refractive index $(n=2.10$ at $\lambda=633 \mathrm{~nm})$, independently of the irradiation conditions [13], suggesting that a steady-state phase is achieved. Moreover, its amorphous character has been ascertained by Raman spectroscopy [12].

The most often used approach to describe track formation is the so-called thermal spike mostly developed by Toulemonde and coworkers [14-17]. It assumes that a hightemperature spike is formed along the ion trajectory and melting can occur. The subsequent rapid cooling leads to amorphization. However, there is evidence that electronic excitation may play a relevant role in the damaging process [18]. Along this line, a nonradiative exciton-decay model has been advanced $[19,20]$ that explains the main features of track formation for $\mathrm{LiNbO}_{3}$ and possibly other oxides. In this model point defects are generated around each ion trajectory through a synergy between the thermal spike and the associated excitation spike. Under multiple ion impacts, the overall defect concentration grows by accumulation, and amorphization occurs at a threshold as a defectdriven phase transition [21] that causes a lattice collapse.

The formation of amorphous tracks by electronic excitation offers very interesting capabilities in nanotechnology. They can be etched (usually by chemical agents) to generate nanopores (or even nanopatterns) that can act as traps for foreign molecular species (sensors) or be refilled with other substances to form nanocomposite materials with novel optical, electrical, and magnetic properties [22-25]. On the other hand, the formation of amorphous layers with a lower refractive index has been advantageously used to fabricate high-confinement optical waveguides [26-28]. They can be formed at the surface or inside the material depending on the stopping power curve. One main advantage of the electronic excitation method is the possibility of tailoring the thickness of the waveguide and of the tunneling barrier that is responsible for a substantial fraction of the optical losses. The new method has been recently used to fabricate ring resonators [29] with nanometric-size confinement of the light.
The purpose of this work has been to investigate the thermal stability of the amorphous tracks and layers generated by swift-ion irradiation in $\mathrm{LiNbO}_{3}$. So far, most information concerning annealing of radiation damage in oxides has been performed in the cases where nuclear mechanisms are dominant (nuclear collision regime) or there is a severe mixing of both electronic and nuclear mechanisms [30-34]. The study presented in this paper has, first, a basic interest in order to get a deeper insight into the annealing behavior of the amorphous regions generated by electronic excitation (electronic excitation regime). For this case, we have shown that the processes involved in the crystal recovery closely resemble those operating during epitaxial regrowth (solid-phase epitaxy). From the applied side our study offers the possibility of modifying the geometry of the nano-structured photonic material produced by the low-fluence swift irradiations and tuning their effective refractive index. On the other hand, the recrystallization of the amorphous buried layers offers a convenient method to tailor the performance parameters of the optical waveguides produced at the sample surface and provides an additional tool for the design of photonic components.

\section{Experimental}

Congruent ( $Z$-cut and $X$-cut) $\mathrm{LiNbO}_{3}$ plates were purchased from Photox. They were irradiated in the 5-MV tandem accelerator installed at the Centro de Microanalisis de Materiales (CMAM-UAM) [35]. Beam current density was lower than $50 \mathrm{nA} / \mathrm{cm}^{2}$ to avoid excessive heating and charging. In fact, by using a high-precision infrared camera (VarioCAM high resolution, Jenoptic Laser, Optik, System $\mathrm{GmbH}$ ) mounted on a $\mathrm{ZnSe}$ viewport it was assessed that the sample temperature was kept below $100^{\circ} \mathrm{C}$. For experiments on isolated tracks, $\mathrm{Br}$ ions at $45 \mathrm{MeV}$ and fluences below $10^{12} \mathrm{~cm}^{-2}$ were mostly used to avoid significant track overlapping. For the annealing of homogeneous amorphous layers, irradiations were performed with $\mathrm{F}$ ions at $20 \mathrm{MeV}$ and moderate fluences, $\sim 10^{14} \mathrm{~cm}^{-2}$. At this energy the maximum electronic stopping power is buried in the sample. Stopping curves for the ions used in this work are shown in Fig. 1. Annealing treatments were performed with two different set-ups in air atmosphere. For the annealing of thick uniform layers, the sample was kept (from 30 minutes to several hours) in a standard tubular Carbolite furnace with an integrated Eurotherm temperature controller assuring thermal uniformity and stability better than $1^{\circ} \mathrm{C}$. On the other hand, for tracks (low fluences of $\mathrm{Br} 45 \mathrm{MeV}$ ), we built a system that consisted of an aluminum solid block with a flat horizontal surface with a cylindrical cartridge heating element inside and a thermocouple located just under the position of the sample. The temperature of the aluminum 


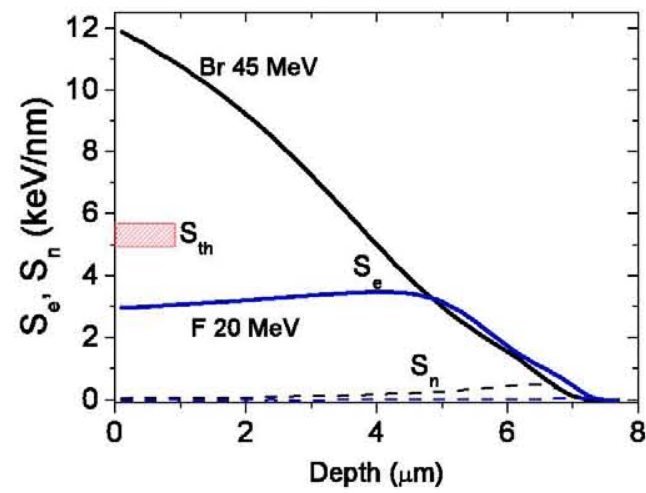

Fig. 1 Electronic and nuclear stopping power curves $\left(S_{e}\right.$ and $\left.S_{n}\right)$ for $\mathrm{Br} 45 \mathrm{MeV}$ and $\mathrm{F} 20 \mathrm{MeV}$ ions in $\mathrm{LiNbO}_{3}$ obtained from SRIM-2003 code. The electronic stopping power threshold for amorphous latent track formation $\left(S_{\text {th }}\right)$ is indicated with a short broad line indicating its approximate value (5-6 keV/nm, velocity dependent)

holder was controlled by an external Eurotherm controller. The aluminum block was heated at the desired temperature. and then the sample was placed quickly on the horizontal hot surface after a few seconds of preheating the sample by holding it close to the hot surface. With the infrared camera it was checked that the final rise time for the temperature on the sample (surface) was in all cases below $3 \mathrm{~s}$, which is considered acceptable. Note that this setup was convenient for some of the short annealings performed at the highest temperatures (for example, $30 \mathrm{sec}$ at $350^{\circ} \mathrm{C}$ ).

The damage recovery during thermal annealing experiments on isolated tracks was monitored by Rutherford Backscattering Spectroscopy in channeling configuration (RBS/C). The sample was mounted on a three-axis goniometer inside the analysis chamber and a beam of ${ }^{4} \mathrm{He}^{+}$ ions at $3 \mathrm{MeV}$ was used as a probe. Backscattered particles were detected by a $16 \mathrm{keV}$ resolution implanted junction detector placed at $170^{\circ}$ from the incident direction.

High-resolution transmission electron microscopy (HRTEM) was used for a few selected samples. Planview samples were prepared for transmission electron microscopy (TEM) examination by dimple-grinding with a Gatan 656 dimpler followed by ion-milling with a Fischione 1010 model until an electron transparent area was obtained in the center of the sample. Transmission electron microscopy studies were done in a JEOL 2010F TEM microscope with a field-emission gun $(200 \mathrm{kV}$ acceleration voltage) and with $0.27 \mathrm{~nm}$ of structural resolution.

For the annealing of homogeneous buried amorphous layers, an optical technique involving the determination of the propagating dark modes through the outer crystalline layer has been preferentially used [36]. For these measurements. light at $\lambda=633 \mathrm{~nm}$ from a He-Ne laser was coupled into the waveguide through a rutile prism. TE and TM polarization geometries were used to observe any possible differences that could be associated to anisotropy induced during swift ion-irradiation or the subsequent annealing. Refractive index profiles were reconstructed following a standard algorithm based on the Wentzel-Kramers-Brillouin (WKB) approximation.

\section{Recrystallization of single amorphous tracks}

\subsection{Kinetics of isothermal annealing}

In principle, the simplest system to investigate the annealing and recrystallization of a region amorphized by swift-ion bombardment is a single amorphous track (in fact, a diluted concentration of them). It has been well ascertained by direct TEM microscopy in lithium niobate $[37,38]$, other dielectric materials, such as sapphire [39] or rutile [40], and semiconductors as silicon [41] that core tracks present a roughly circular shape, regardless of the crystalline symmetry, and essentially amorphous structure. This is, indeed, consistent with the initial round dips generated [42] by chemical etching in $\mathrm{LiNbO}_{3}$. Although it is known that the amorphized fraction measured by RBS/C decreases after thermal treatments [43], the effect of annealing on individual tracks has not been directly investigated. In this work, a number of systematic isothermal annealing experiments have been performed on Z-cut samples irradiated at $\mathrm{RT}$ with $\mathrm{Br}$ at $45 \mathrm{MeV}$ and followed by monitoring the extension of the amorphized area by RBS/C with He probing ions. The objective is to subject the crystal to irradiation with ions having a stopping power clearly above the amorphization threshold, which is around 5-6 keV at the surface (see Fig. 1), so that continuous well-defined tracks are generated. Moreover, a low fluence irradiation $\left(<10^{12} \mathrm{~cm}^{-2}\right)$ was used to assure a small enough overlapping of the tracks. In principle, the full-depth amorphization profile can be measured by RBS/C. However, the method is particularly reliable to measure the extension of the amorphized area at the sample surface, where no significant lattice dechanneling occurs. The aim is to measure the shrinking rate of the track cross-sections at the sample surface as a function of annealing time for several temperatures. Figure 2 shows as an example one set of RBS/C spectra corresponding to the annealing evolution for a temperature of $275^{\circ} \mathrm{C}$ and a fluence of $5 \times 10^{11} \mathrm{~cm}^{-2}$. The kinetics of isothermal annealing on the fractional disordered area at several temperatures in the range $250-350^{\circ} \mathrm{C}$ is illustrated in Fig. 3a corresponding to an irradiation fluence of $\phi=3 \times 10^{11} \mathrm{~cm}^{-2}$. Similar results for samples irradiated at $\phi=5 \times 10^{11} \mathrm{~cm}^{-2}$ and $\phi=1 \times 10^{12} \mathrm{~cm}^{-2}$ are shown, respectively, in Figs. $3 \mathrm{~b}$ and $3 \mathrm{c}$. The insets in the figures illustrate the damage morphology by showing a random Monte Carlo distribution of disks, having a radius of around $4.5 \mathrm{~nm}$ (as estimated from the results of Fig. 3a for the lowest fluence), with the density corresponding to the fluence. They 


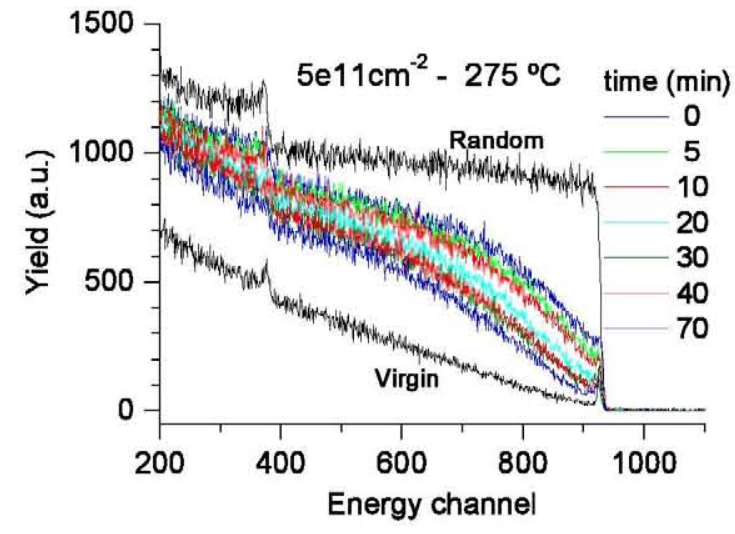

Fig. $2 \mathrm{RBS} / \mathrm{C}$ spectra measured, with an ion beam of $\mathrm{He} 3 \mathrm{MeV}$ as a probe, for the case of an irradiated sample with a fluence of $5 \mathrm{e} 11 \mathrm{~cm}^{-2}$ (time $=0$ ) and annealed in air at $275^{\circ} \mathrm{C}$ in six steps indicated in the figure labels (time in minutes), giving rise to a progressive decrease in the measured yield

visualize how the track overlapping has clearly increased with fluence. From these data it is clear that the overall track cross-section decreases with annealing time, being slightly dependent on the irradiation fluence (the rate appears to decrease with increasing fluence). As we will see later, it appears more meaningful to plot the evolution of the track radius with annealing time. To this end, we have, initially, assumed that tracks are fully independent (not overlapped) and so the disordered area is simply the cross-section of a track multiplied by the impact density. It is also assumed that the track cross-sections maintain the circular shape during the annealing as expected for tracks along the Z-axis due to the axial crystal symmetry. Using these approximations, one readily obtains the evolution of (core) track radius with annealing times for the same temperatures and fluences as in Fig. 2. The results are shown in Fig. 4. Now, it becomes clear that the velocity of radial decrease, $v=d R / d t$, presents two well-defined linear regimes. There is a fast initial stage (I) until the radius shrinks to around or below $2.5 \mathrm{~nm}$, and, then, a stage II sets in where shrinking proceeds at a slower rate. Note that in the main stage $\mathrm{I}$, the radial velocity at $350^{\circ} \mathrm{C}$ amounts to about $7 \mathrm{~nm} / \mathrm{min}$.

\subsection{Stage I: role of irradiation fluence}

Most reliable analysis of the kinetic behavior can be performed for the main and faster annealing stage I. From a comparison of the plots in Fig. 4, it can be appreciated that, for such a stage, the radial velocity $v$ slightly decreases on increasing fluence. One should note from the insets that some overlapping already exists for $5 \times 10^{11} \mathrm{~cm}^{-2}$ and it becomes serious for $1 \times 10^{12} \mathrm{~cm}^{-2}$. Therefore one should be cautious with the description of a proper track radius for the highest fluences. Nevertheless, keeping the analysis in terms of the (effective) track radius, Fig. 5 illustrates the comparison of the velocities for the three investigated fluences. The
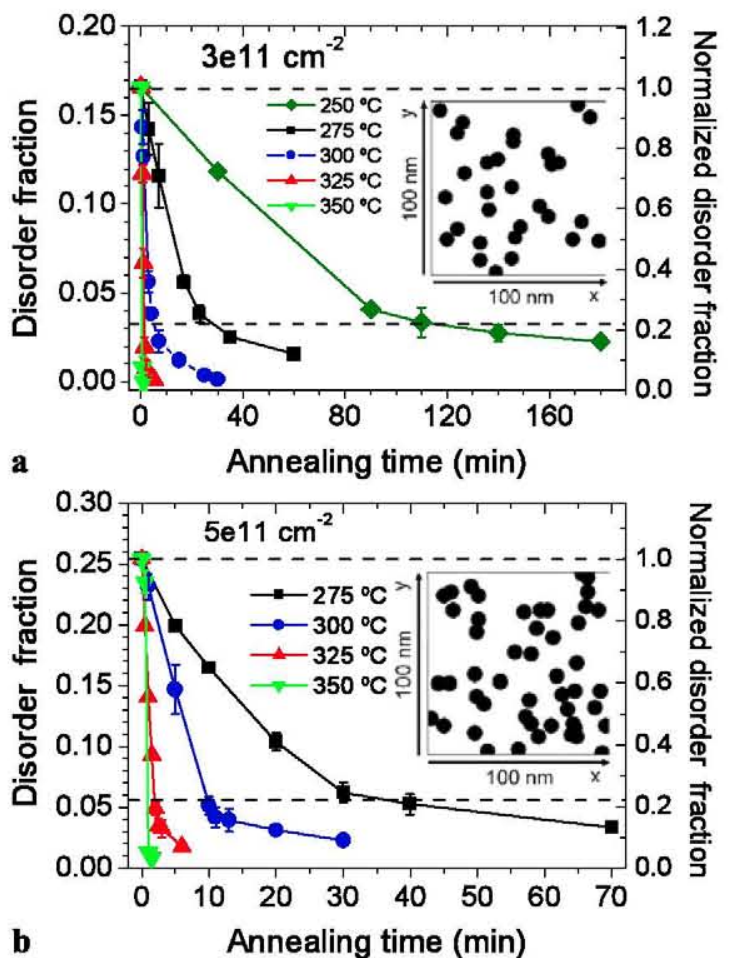

Fig. 3 Disordered fraction obtained from RBS/C versus annealing time for the fluences (a) $3 \times 10^{11} \mathrm{~cm}^{-2}$, (b) $5 \times 10^{11} \mathrm{~cm}^{-2}$, and (c) $1 \times 10^{12} \mathrm{~cm}^{-2}$, and for the temperatures 250, 275, 300, 325, and $350^{\circ} \mathrm{C}$, as indicated in the figures labels. The insets show schematic illustrations of the covered area, for each of the three fluences, simulated with random impacts of estimated track radius of $4.4 \mathrm{~nm}$

reason of the dependence found is likely associated to the reduction of the crystalline-amorphous interface due to track overlapping. We will come back to this point later.

Since the recrystallization process appears to be thermally activated, we have plotted in Fig. 6 the radial velocity data for stage $\mathrm{I}$ as a function of temperature using the Arrhenius-type plot, $v=v_{0} \exp \{-\varepsilon / k T\}$. All the data reasonably fit the Arrhenius dependence with an activation energy between $\varepsilon=1.46 \mathrm{eV}$ and $\varepsilon=1.58 \mathrm{eV}$, slightly dependent on fluence. Since the differences are within the error range, one may conclude that the activation energy is not substantially modified by fluence. Possible recrystallization 

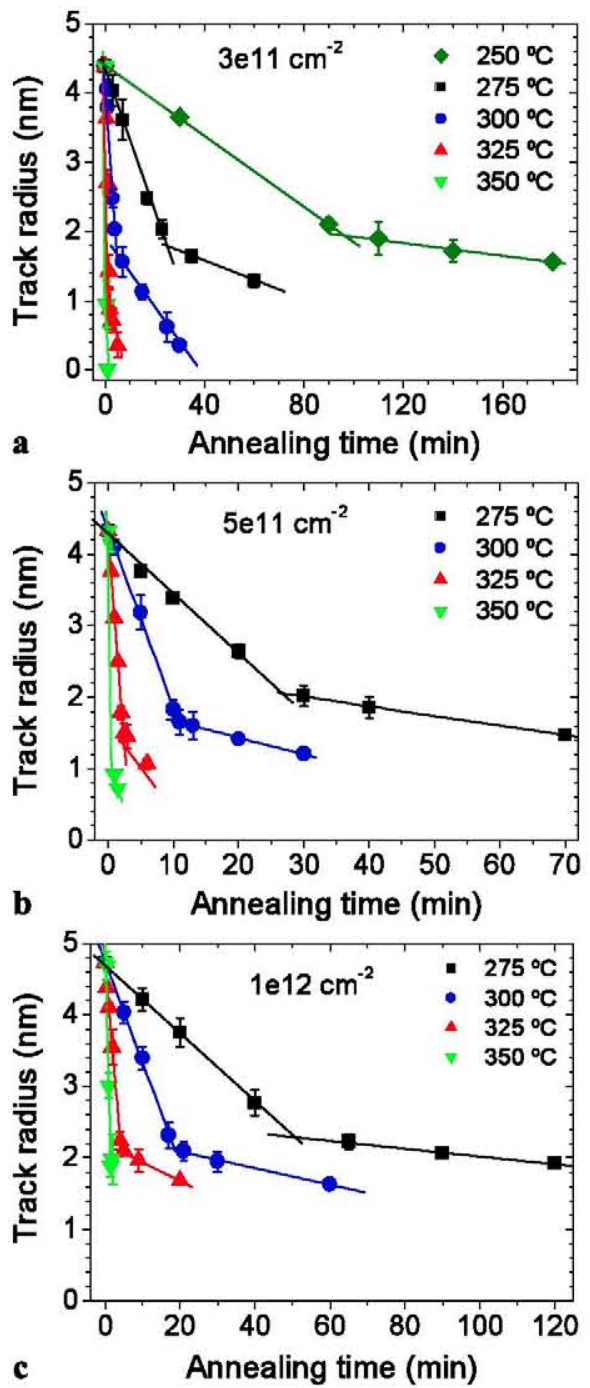

Fig. 4 Evolution of the track radius with annealing time for the fluences (a) $3 \times 10^{11} \mathrm{~cm}^{-2}$, (b) $5 \times 10^{11} \mathrm{~cm}^{-2}$, and (c) $1 \times 10^{12} \mathrm{~cm}^{-2}$, and for the temperatures $250,275,300,325$, and $350^{\circ} \mathrm{C}$, as indicated in the figures labels. The track radius is obtained for each case from the corresponding disordered fraction (shown in Fig. 3) as described in the text assuming a linear dependence of the cross-section of a track multiplied by the impact density

mechanisms accounting for this behavior will be discussed below.

\subsection{Slow annealing stage II}

One interesting output of our annealing experiments is the existence of a slower annealing stage (II) near the end of the recovery process, once the track radius has reached a value around or smaller than $2.5 \mathrm{~nm}$. The data cover a limited number of data points with a higher relative error, and so their analysis cannot be as conclusive as for the main annealing stage. Anyhow, the shrinking rate of stage II is also roughly linear and clearly smaller than that for stage I.

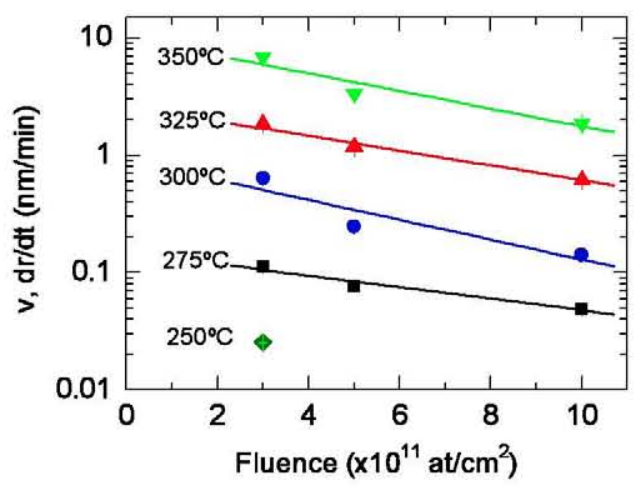

Fig. 5 Radial velocity of recrystallization as a function of fluence for the annealing temperatures indicated in the figure

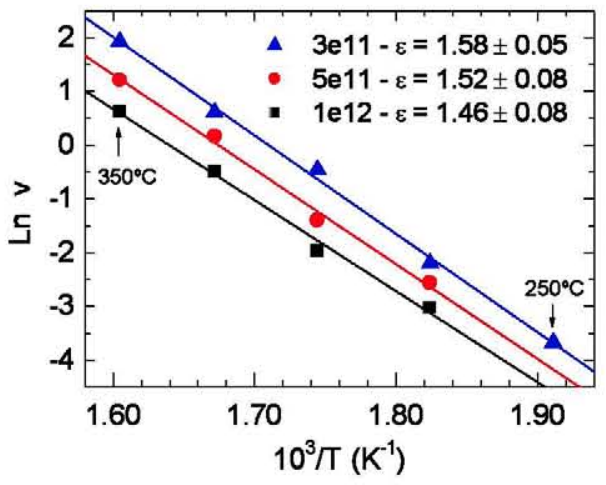

Fig. 6 Arrhenius-type plot of the radial velocity data for the stage I for the three studied fluences indicated in the figure with their corresponding activation energies

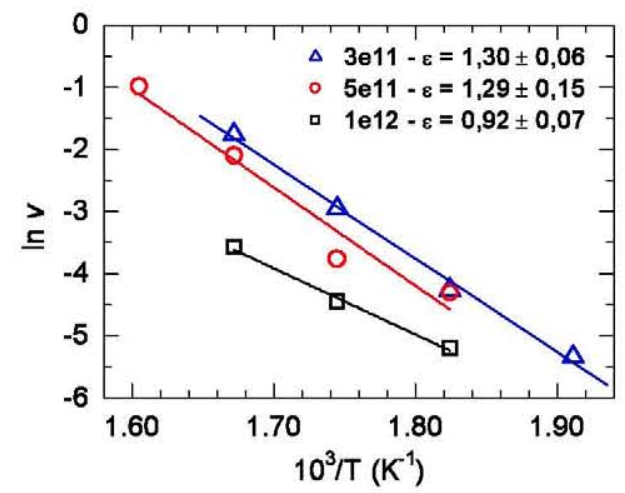

Fig. 7 Arrhenius-type plot of the radial velocity data for the stage II for the three studied fluences indicated in the figure with their corresponding activation energies

Moreover, it is thermally activated as inferred from the $\mathrm{Ar}$ rhenius plot shown in Fig. 7. One sees that the radial velocities are a factor 2-3 smaller than for the main linear annealing stage, Fig. 6 . The data show large dispersion, although they approximately obey an Arrhenius-type law. The activation energies (between $0.92 \mathrm{eV}$ and $1.30 \mathrm{eV}$ ) are not very different from those for stage I. In order to check that the 


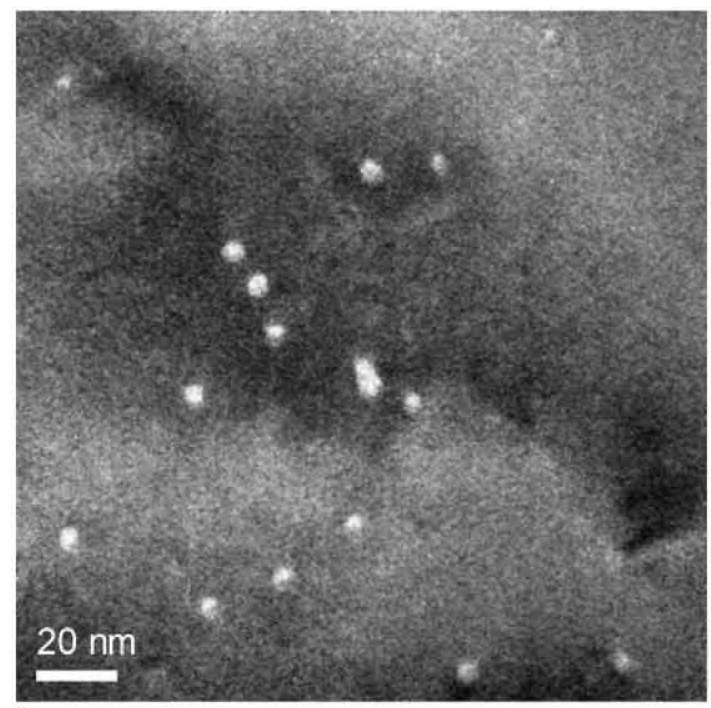

a

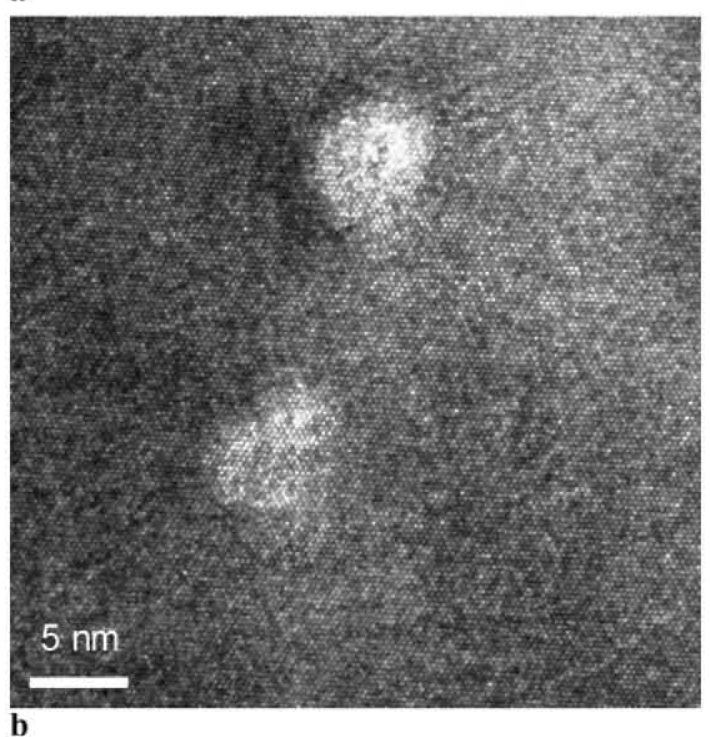

b

Fig. 8 TEM images (two scales) of a $\mathrm{LiNbO}_{3}$ sample irradiated with $\mathrm{Br} 45 \mathrm{MeV}$ ions at a fluence of $3 \times 10^{10} \mathrm{at} / \mathrm{cm}^{2}$ after annealing in air for $30 \mathrm{~min}$ at $275^{\circ} \mathrm{C}$. (a) Several circular tracks can be observed; (b) high resolution image shows that the radius of the tracks (of about $2.5 \mathrm{~nm}$ ) are consistent with data obtained from RBS/C

structure of the track at the inflexion point separating stages I and II is not anomalous, TEM images have been obtained at that point, and two of them are shown in Fig. 8a, b. The shape is still circular, and the radius is fairly consistent with the RBS/C data, although one should be cautious due to possible electron-induced recrystallization $[44,45]$.

\section{Physical mechanisms}

It appears reasonable to start, first, with the recovery of individual amorphous tracks, where most complete information has been gathered. Moreover, they appear as the individual ingredients forming the homogeneous amorphous layers. In principle, we will here focus on the more reliable data for stage I. As in other solid-phase epitaxy processes [46], one may assume that the recrystallization occurs at the crystalline-amorphous boundary. In fact, all data have been interpreted in accordance with this view considering that, during the annealing, the amorphous cores keep a circular cross-section with decreasing radius. On the other hand, it is expected that the basic $\mathrm{Nb}-\mathrm{O}_{6}$ octahedral unit stay essentially bound (rigid) in both crystalline and amorphous phases. The amorphous phase should only imply some random reordering of the crystalline octahedral arrangement. Therefore, one may think that the octahedra lying in the amorphous phase close to the crystalline boundary are those that may thermally accommodate into the crystalline structure and move the boundary. In other words, the rate of recrystallization is proportional to the number of octahedral sites at the boundary, i.e., to the perimeter of such a boundary. This view implies that the octahedral reordering is a first-order local process that does not depend on the long-range diffusion of point defects as vacancies or interstitials. In other words, the octahedra at the boundary sites behave independently of each other and have a definite frequency $\eta$ for recovery (reorienting). This readily accounts for the constancy of the radial recrystallization velocity, and it is in accordance with more general formulations of solidstate epitaxy or epitaxial regrowth such as the KolmogorovJohnson-Mell-Avrami (KJMA) model [47, 48]. The linear boundary motion or constant recrystallization rate has also been invoked in the annealing of ion-beam irradiated $\mathrm{ABO}_{3}$ oxides [30-34], mostly for the elastic nuclear collision regime.

The reorientation frequency should be an Arrhenius function of temperature $v=v_{0} \exp \{-\varepsilon / k T), \varepsilon$ being a certain energy barrier to be overcome for recovery. This is in accordance with our assumption that recovery is a local process as expected for a first-order phase transition (no long-range atomic motion or diffusion is involved). Then, the kinetics of recovery for the cross-section area $\sigma$ of a single track would be

$\frac{d \sigma}{d t}=-v(2 \pi R) \delta=-\left(2 v \pi^{1 / 2}\right) \sigma^{1 / 2}$

and, for the track radius $R$,

$\frac{d R}{d t}=-v \delta$

where $v=v \delta$ is the radial velocity of the track boundary, and $\delta$ some average value for the lattice parameter. In other words, one expects a linearly decreasing law for the time evolution of the radius, $R(t)=R_{0}-v \delta t$, in accordance with the experimental data points, Fig. 4. For the main linear stage I of annealing, the value of the frequency $v$ amounts to around $10^{-3} \mathrm{~s}^{-1}$ at $275^{\circ} \mathrm{C}$. Note that it is a rather low 
value that may correspond to some rotational frequency of the octahedra. Taking into account that the octahedra are anchored by the $\mathrm{O}$ corners, it is not yet clear how this rotation may occur. The possible role of point defects such as $\mathrm{O}$ vacancies should also be elucidated. It is expected that the reorientation process would involve the overcoming of certain energy barriers and so its rate should be thermally activated. In fact, Fig. 6 shows that, in spite of the limited temperature range, the data for $V$, and thus for $v$, present a satisfactory fit to the Arrhenius plot. The energy barrier for recovery is in the range $1.46-1.58 \mathrm{eV}$, which appears reasonable for atomic jump processes. From the Arrhenius equation, $v=v_{0} \exp \{-\varepsilon / k T\}$, one obtains a preexponential factor of around $\nu_{0} \sim 10^{9}-10^{11} \mathrm{~s}^{-1}$.

\subsection{Role of irradiation fluence}

We have advanced in Sect. 4 that the decrease observed in the effective boundary velocity on increasing fluence should, likely, be due to a reduction in the extension of the crystalline-amorphous boundary (i.e., in the density of recristallization sites). This argument may be put in a more quantitative form by using a simple Monte Carlo approach to describe the distribution of the amorphous sections of the tracks (disks) in a transversal plane. The overall perimeter of the distribution can be estimated from the morphologies illustrated in the insets of Fig. 3 by using a simple Monte Carlo code. The obtained dependence on track density (fluence) is displayed in Fig. 9. One sees an initial stage wherein the perimeter per track is constant, as expected for fully isolated tracks. Around a certain critical fluence $\left(\approx 3 \times 10^{11} \mathrm{~cm}^{-2}\right)$ the normalized perimeter decreases with increasing fluence as a consequence of the track overlapping. As expected, the critical fluence, at which a change in behavior is observed, roughly corresponds to the impact density leading to optimum disk packing (coverage). In accordance with this view, the recrystallization velocity should decrease with increasing irradiation fluence. To check this assumption, we have renormalized the recrystallization velocities in relation to the effective perimeter of the crystalline-amorphous boundary, Fig. 10. One sees that the accordance with experiment has improved, but still there remains a significant discrepancy. One possible effect, not considered so far, has to do with the role of overlapping defective halos around the track amorphous core [49] that may hinder the recristallization process. In fact, as shown in Fig. 9, the agreement with experiment can be achieved by artificially increasing the radius of the track core from $4.5 \mathrm{~nm}$ to around $5.5 \mathrm{~nm}$ or even $6.5 \mathrm{~nm}$. The physical value of this assumption has to be explored in future work.

\subsection{Slow annealing stage (II)}

The occurrence of a slower annealing stage at the end of the recovery process cannot be well understood with the

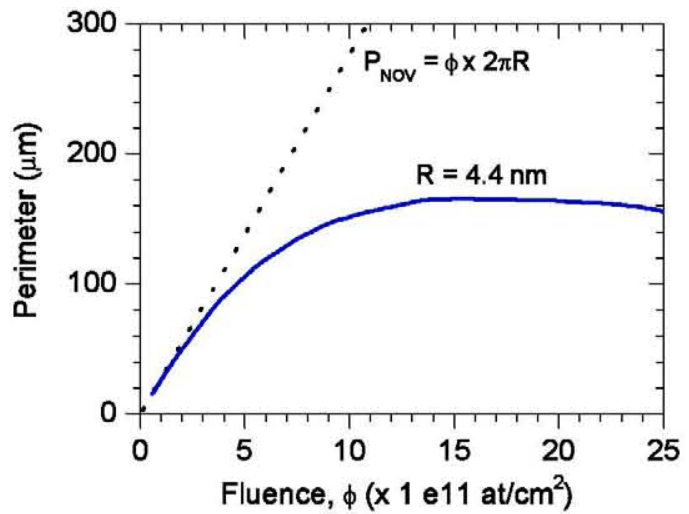

Fig. 9 Overall perimeter as a function of fluence of a random track distribution of radius $R=4.4 \mathrm{~nm}$ numerically simulated with a Monte Carlo code (solid line). The linear dependence assuming nonoverlapping tracks is shown as reference (dotted line)

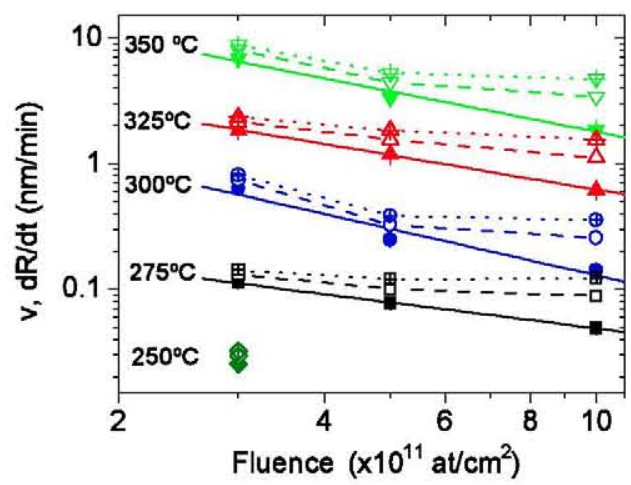

Fig. 10 Recrystallization velocity for the temperatures indicated in the figure renormalized to the effective perimeter for two different radii (4.4 and $5.5 \mathrm{~nm}$ ). The solid symbols and lines are the experimental data also shown in Fig. 5. The open symbols correspond to the renormalized data. Dashed line corresponds to a radius of $4.4 \mathrm{~nm}$, and dotted line to a radius of $5.5 \mathrm{~nm}$

available information. In fact, one may consider that for track radii around or below $2 \mathrm{~nm}$, the discrete nature of the crystal lattice should be taken into account. On the other hand, the normalized data also shown in Figs. 3a, 3b, and $3 \mathrm{c}$ (right axis) showing that the change from stage I to stage II seems to occur at a disorder percentage of about $25 \%$, independently of the fluence, may suggest a residual track core with a more stable structure, although this is difficult to justify unless additional experiments are carried out. It could be also argued that the recovered crystalline surrounding (halo) is more and more defective for decreasing track radii causing an enhancement of the channeled RBS yield over that one for the amorphous core. The use of an independent technique such as HRTEM observations and the appropriate data analysis would help to solve the problem. 


\section{Recrystallization of homogeneous amorphous layers}

According to current models of swift ion-beam amorphization, the formation of homogeneous amorphous layers results from the overlapping and accumulation of single tracks [50]. Therefore, one would expect coherent results for the annealing kinetics of both, tracks and layers. Optical (dark mode) techniques are particularly suitable to investigate amorphous layers that have been produced under moderate fluences inside the sample leaving crystalline the outermost surface layer. In this case the measurement of the propagating optical modes provides accurate and detailed information on the depth damage profile of the irradiated crystal. Buried layers have been produced [36] by irradiation with $\mathrm{F}$ ions at $20 \mathrm{MeV}$, whose stopping power curve as a function of depth presents a maximum inside the sample (below the surface, see Fig. 1). In this case a crystalline layer appears at the surface limited by the amorphous buried layer acting as an optical confinement medium because of its lower refractive index, as illustrated in the data of Fig. 11. The amorphous nature of the buried damaged layer is inferred from the measured refractive index profiles for both the TE and TM modes that decrease down to the amorphous value, 2.10 (at $633-\mathrm{nm}$ wavelength) on reaching the outer amorphous boundary. This conclusion is also confirmed by the micro-Raman spectra showing the stacking of crystalline and amorphous layers [36]. Our optical method yields the depth refractive index profile within the crystalline (waveguiding) layer and so provides information on the motion of the crystalline-amorphous boundary.

In order to monitor in detail the kinetics of the recrystallization process, the samples irradiated with $\mathrm{F}(20 \mathrm{MeV})$ at $4 \times 10^{14} \mathrm{~cm}^{-2}$ have been kept at $350^{\circ} \mathrm{C}$ for different isothermal annealing times in air up to $10 \mathrm{~h}$. The refractive index profiles obtained after different times for a $Z$-cut sample are shown in Figs. 11a and $11 \mathrm{~b}$ for ordinary and extraordinary polarization, respectively. These profiles determine the high index values in the outer crystalline layer, wherein optical modes are propagated, and the index jump at the outer layer of the amorphous layer. Now, one clearly observes the inward motion of that boundary and the corresponding widening of the waveguide at the surface. Figure 12 shows the thickness of the outer crystalline layer (waveguide) as a function of annealing time. The dependence is essentially linear as found for the annealing of individual tracks, and the linear recrystallization velocity is near $3 \mathrm{~nm} / \mathrm{min}$, i.e., of the same order of magnitude as the radial velocity for circular tracks. The same phenomenological model developed for isolated tracks can be applied to the recovery of full homogeneous layers. For a layer of initial thickness $h$, the kinetics is a simple extension of (2), i.e.,

$$
\frac{d h}{d t}=-v \delta
$$
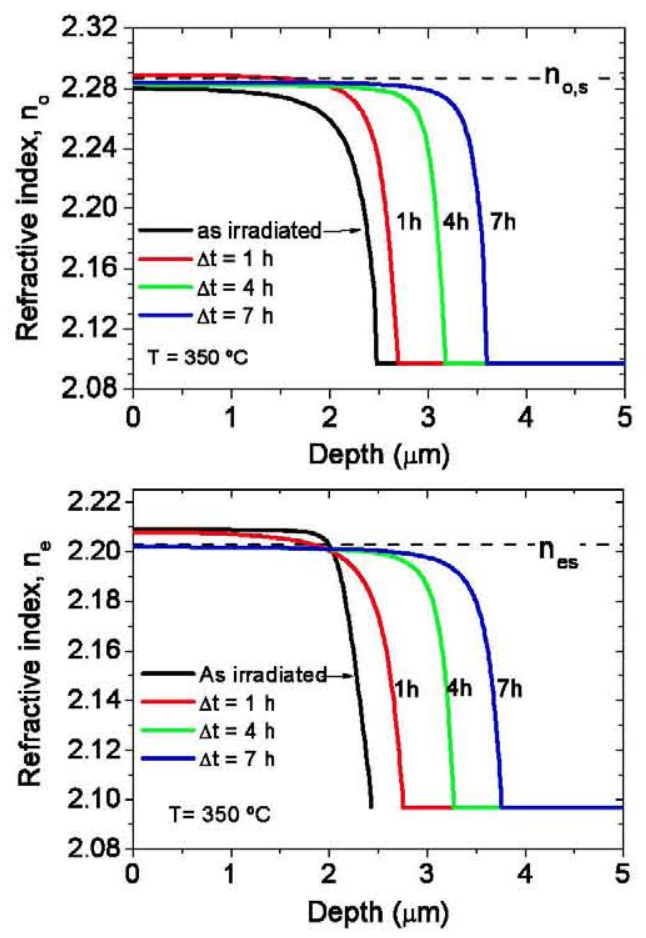

Fig. 11 Refractive index (ordinary and extraordinary) profile evolution obtained after several steps $(1,4$, and $7 \mathrm{~h})$ of annealing in air at $350^{\circ} \mathrm{C}$ for a waveguide produced with $\mathrm{F} 20 \mathrm{MeV}$ irradiation at a fluence of $4 \times 10 \mathrm{~cm}^{-2}$

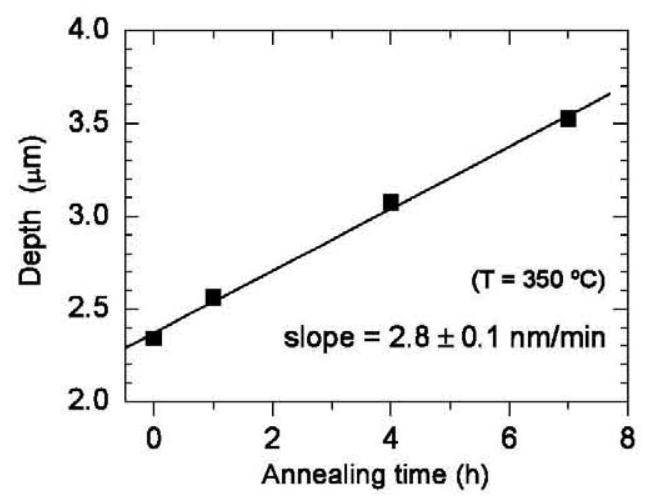

Fig. 12 Evolution of the crystalline-amorphous boundary position (depth) with annealing time at $350^{\circ} \mathrm{C}$ for the waveguide whose refractive index profile is shown in Fig. 11

predicting a linear motion of the boundary. However, more work is still necessary for a meaningful comparison of the kinetics of recrystallization of single tracks and amorphous layers.

\section{Comparison with the recrystallization of amorphous layers generated by elastic nuclear collision damage}

In relation to our work, one should pay special attention to extensive work on perovskites and related oxides, using 
both, light and heavy ions, mostly for applications to the storage and immobilization of radioactive waste [30-34]. The study has concentrated on the nuclear stopping regime or a mixed regime with a substantial contribution of nuclear collisions to the ion losses. In this case, the incident ion generates primary and secondary atomic displacements (Frenkel pairs) leading to displacement cascades, whose accumulation finally causes an amorphous stage. The overall process can be simulated by advanced Monte Carlo codes. Although the initiation of a definite amorphous phase is difficult to ascertain, it is generally accepted that it occurs for fluences around or slightly above $1 \mathrm{dpa}$ (displacements per atom). It appears to be a gradual (monotonic) process resulting from the accumulation of successive displacement spikes. Moreover, the detailed structure of such an amorphous phase is also controversial and maybe not unique.

In the case of electronic damage, each individual ion impact produces either isolated defects for electronic stopping powers below a well-defined threshold or an amorphous track above such threshold. Unfortunately, the atomic structure of the defects created by electronic excitation is not known, and more research along the lines developed in [5] is required. Anyhow, for electronic excitation, amorphization already occurs at the individual impact (track) level. This amorphization process appears to result from a phase transformation (either melting or a defect-driven transition) and has definite physical properties (refractive index, electronic properties, ...). This is a key difference with nuclear amorphization. On the other hand, the overall electronic damage after a multiimpact irradiation also results from the accumulation of the individual tracks. A detailed Monte Carlo analysis of the electronic damage morphology has been recently reported [50]. One main difficulty when trying to compare the recovery of the amorphous regions caused by nuclear collision or electronic damage is the fact that there are not clear-cut experiments where amorphization is exclusively produced by collision processes. In fact, experiments with light ions where electronic and nuclear damage regions are well separated hardly cause amorphization (except at very high fluences). So, the annealing data show a large variety of recovery temperatures that cannot be safely associated to an amorphous phase but to intermediate stages of disorder. On the other hand, many other experiments performed with heavy ions have been done at energies where electronic and nuclear effects are completely intermixed. Moreover, one cannot ignore the role of the implanted ions reaching concentrations of $10^{-2}-10^{-3}$ on the amorphization process as it has been clearly revealed [29] (e.g., $\mathrm{Pb}$ at $E<1 \mathrm{MeV}$ ). Anyhow, the available data depend strongly on ion and fluence. On the other hand, the structure of the phases can be quite different. For electronic damage, one can imagine some kind of mosaic structure made up of dislocated octahedra.

In particular, we can compare our data with those obtained [30] in some perovskites after $0.5 \mathrm{MeV}$ irradiation with $\mathrm{Pb}$. They clearly show by monitoring the recovery with RBS/C that amorphous regions recrystallize epitaxially with motion of the boundary toward the surface. The recrystallization temperatures are similar as those corresponding to our experiments. Constant isothermal recrystallization rates are of the same order as those found in our experiments, e.g., around $0.1 \mathrm{~nm} / \mathrm{s}$ at $300^{\circ} \mathrm{C}$ and $0.3 \mathrm{~nm} / \mathrm{s}$ at $350^{\circ} \mathrm{C}$. One may suspect that the situation could be strongly influenced by electronic amorphization. On the other hand, activation energies are around $1 \mathrm{eV}$. All those similarities may suggest that the two processes cause a similar structure for the damage. This is consistent with the idea that basic transition metal octahedra are quite stable and amorphization can be understood as a random ordering of such octahedra.

\section{Summary and conclusions}

In this work we have provided systematic data on the recrystallization kinetics of single amorphous tracks and homogeneous layers. The data for single tracks reveal that annealing is governed by interface processes as for solid-state epitaxy. The velocity of the crystalline-amorphous boundary moves at constant velocity $V \sim 3 \mathrm{~nm} / \mathrm{min}$ (at $350^{\circ} \mathrm{C}$ ) in a main annealing stage but slows down for track radii of around $2 \mathrm{~nm}$. The velocity is thermally activated in accordance with an Arrhenius-type dependence and an activation energy around $1.5 \mathrm{eV}$ for the main initial stage. For track radii smaller than $\sim 2 \mathrm{~nm}$, the track shrinkage process slows down suggesting that other mechanisms should be taken into account. For homogeneous amorphous layers, the data can be described within the same physical process and provide consistent data with those obtained for individual tracks.

The results may have relevant implications for a number of applications. The control of track radii by suitable annealing treatments can be used to tailor the diameter of the nanopores after suitable chemical etching. Moreover, the control of track radii allows us to tailor the dielectric constant and refractive index of the outer irradiated layer and so the possibilities for integrated optics devices. Also, for the photonics field, the results found for homogeneous layers allow for an adequate control of the thickness of the outer waveguide layer and even the sharpness of the waveguide boundary [25]. Last but not least, the annealing data are, indeed, very relevant in connection with the problem of radioactive waste disposal.

Acknowledgements We acknowledge financial support from the Spanish Ministry of Science and Innovation for the project MAT-200806794-C03-03.

\section{References}

1. P.D. Townsend, P.J. Chandler, L. Zhang, Optical Effects of Ion Implantation (Cambridge University Press, Cambridge, 1994) 
2. M. Nastase, J.W. Mayer, J.H. Hirvonen, Ion-Solid Interactions: Fundamental and Applications (Cambridge University Press, Cambridge, 1996)

3. F. Agulló-López, R.C. Catlow, P.D. Townsend, Point Defects in Materials (Academic Press, New York, 1984)

4. A. Rivera, J. Olivares, G. García, J.M. Cabrera, F. Agulló-Rueda, F. Agulló-López, Phys. Status Solidi A 206, 1109 (2009)

5. C.S. Schnohr, P. Kluth, A.P. Byrne, G.J. Foran, M.C. Ridway, Phys. Rev. B 77, 073204 (2008)

6. S.M.M. Ramos, R. Brenier, B. Canut, G. Fuchs, P. Thevenard, M. Trilleux, A. Meftah, M. Toulemonde, J. Appl. Phys. 77, 2952 (1995)

7. B. Canut, S.M.M. Ramos, R. Brenier, P. Thevenard, J.L. Loubet, M. Toulemonde, Nucl. Instrum. Methods Phys. Res., Sect. B, Beam Interact. Mater. Atoms 107, 194 (1996)

8. F. Thibaud, J. Cousty, E. Balanzat, S. Bouffard, Phys. Rev. Lett. 67, $1582(1991)$

9. J. Olivares, A. García-Navarro, G. García, A. Méndez, F. AgullóLópez, Appl. Phys. Lett. 89, 071923 (2006)

10. P. Kluth, C.S. Schnohr, O.H. Pakarinen, F. Djurabekova, D.J. Sprouster, R. Giuliani, M.C. Ridway, A.P. Byrne, C. Trautmann, D.J. Cookson, K. Nordlund, M. Toulemonde, Phys. Rev. Lett. 101, $175503(2008)$

11. N. Itoh, D.M. Duffy, S. Khakshouri, A.M. Stoneham, J. Phys., Condens. Matter 21, 474205 (2009)

12. J. Olivares, G. García, F. Agulló-López, F. Agulló-Rueda, A. Kling, J.C. Soares, Appl. Phys. A 81, 1465 (2005)

13. G. García, F. Agulló-López, J. Olivares-Villegas, A. GarcíaNavarro, J. Appl. Phys. 99, 1 (2006)

14. Z.G. Wang, C. Dufour, E. Paumier, M. Toulemonde, J. Phys., Condens. Matter 34, 6733 (1994)

15. G. Szenes, Phys. Rev. B 51, 8026 (1995)

16. M. Toulemonde, W. Assman, C. Dufour, A. Meftah, F. Studer, C. Trautmann, in Ion Beam Science: Solved and Unsolved Problems, ed. by P. Sigmund (The Royal Danish Academy of Sciences and Letters, Copenhagen, 2006), p. 263

17. A. Kamarou, W. Wesch, E. Wendler, A. Undisz, M. Rettenmayr, Phys. Rev. B 73, 184107 (2007)

18. N. Itoh, A.M. Stoneham, Materials Modification by Electronic Excitation (Cambridge University Press, Cambridge, 2001)

19. F. Agulló-López, A. Méndez, G. García, J. Olivares, J.M. Cabrera, Phys. Rev. B 74, 174109 (2006)

20. A. Rivera, A. Méndez, G. García, J. Olivares, J.M. Cabrera, F. Agulló-López, J. Lumin. 128, 703-707 (2008)

21. H.J. Fecht, Nature (London) 356, 133 (1992)

22. R. Spohr, in Ion Tracks and Microtechnology: Basic Principles and Applications, ed. by K. Bethge (Vieweg, Braunschweig, 1990)

23. M. Toulemonde, C. Trautmann, E. Balanzat, K. Horjt, A. Weidinger, Nucl. Instrum. Methods Phys. Res., Sect. B, Beam Interact. Mater. Atoms 216, 1 (2004)

24. J. Chen, R. Könenkamp, Appl. Phys. Lett. 82, 4782 (2003)

25. M. Sima, I. Enculescu, C. Trautmann, R. Neumann, J. Optoelectron. Adv. Mater. 6, 124 (2004)

26. J. Olivares, G. García, A. García-Navarro, F. Agulló-López, O. Caballero, A. García-Cabañes, Appl. Phys. Lett. 86, 183501 (2005)

27. J. Olivares, M.L. Crespillo, O. Caballero-Calero, M.D. Ynsa, A. García-Cabañes, M. Toulemonde, C. Trautmann, F. Agulló-López, Opt. Express 17, 24176 (2009)
28. C.A. Merchant, P. Scrutton, S. García-Blanco, C. Hnatovski, R.S. Taylor, A. García-Navarro, G. García, F. Agulló-López, J. Olivares, A.S. Helmy, J.S. Aitchison, IEEE J. Quantum Electron. 45(4), 373 (2009)

29. A. Majkic, M. Koechlin, G. Poberaj, P. Günter, Opt. Express 16, 8769 (2008)

30. C.W. White, L.A. Boatner, P.S. Sklad, C.J. McHargue, J. Rankin, G.C. Farlow, M.J. Aziz, Nucl. Instrum. Methods Phys. Res., Sect. B, Beam Interact. Mater. Atoms 32, 11 (1988)

31. A. Meldrum, S.J. Zinkle, L.A. Boatner, R.C. Ewing, Phys. Rev. B 59, $3981(1998)$

32. Z. Zhang, I.A. Ruskova, W.K. Chu, Nucl. Instrum. Methods Phys. Res., Sect. B, Beam Interact. Mater. Atoms 136-138, 404 (1998)

33. W.J. Weber, Nucl. Instrum. Methods Phys. Res., Sect. B, Beam Interact. Mater. Atoms 166-167, 98 (2000)

34. A. Meldrum, L.A. Boatner, W.J. Weber, R.C. Swing, J. Nucl. Mater. 300, 242 (2002)

35. www.cmam.uam.es

36. J. Olivares, A. García-Navarro, G. García, F. Agulló-López, F. Agulló-Rueda, A. García-Cabañes, M. Carrascosa, J. Appl. Phys. 101, 33512 (2007)

37. B. Canut, R. Brenier, A. Meftah, P. Moretti, S. Ould Salem, M. Pitaval, S.S.M. Ramos, P. Thevenard, M. Toulemonde, Radiat. Eff. Defects Solids 136, 307 (1995)

38. S.S.M. Ramos, B. Canut, M. Ambri, C. Clement, E. Doorhye, M. Pitaval, P. Thevenard, M. Toulemonde, Nucl. Instrum. Methods Phys. Res., Sect. B, Beam Interact. Mater. Atoms 107, 254 (1996)

39. S.S.M. Ramos, N. Bonardi, B. Canut, S. Della-Negra, Phys. Rev. B 57, 189 (1998)

40. K. Awazu, X. Wang, M. Fujimaki, T. Komatsubara, T. Ikeda, Y. Ohki, J. Appl. Phys. 100, 044308 (2006)

41. A. Dunlop, G. Jaskierowicz, S. Della-Negra, Nucl. Instrum. Methods Phys. Res., Sect. B, Beam Interact. Mater. Atoms 146, 302 (1998)

42. M. Crespillo, M. Otto, A. Muñoz-Martin, J. Olivares, F. AgullóLópez, M. Seibt, M. Toulemonde, C. Trautmann, Nucl. Instrum. Methods Phys. Res., Sect. B, Beam Interact. Mater. Atoms 267, 1035 (2009)

43. G.G. Bentini, M. Bianconi, M. Chiarini, L. Correra, C. Sada, P. Mazzoldi, N. Argiolas, M. Bazzan, R. Guzzi, J. Appl. Phys. 92, 6477 (2002)

44. A. Benyagoub, A. Audren, L. Thomé, F. Garrido, Appl. Phys. Lett. 89, 241914 (2006)

45. Y. Zhang, J. Lian, C.M. Wang, W. Jiang, R.C. Ewing, W.J. Weber, Phys. Rev. B 72, 094112 (2005)

46. I. Markov, Crystal Growth for Beginners, 2nd edn. (World Scientific, Singapore, 2003)

47. W. Johnson, R. Mehl, Trans. Metall. Soc. AIME 135, 416 (1939)

48. M. Avrami, J. Chem. Phys. 7, 1103 (1939)

49. A. Rivera, J. Olivares, M.L. Crespillo, G. García, M. Bianconi, F. Agulló-López, Nucl. Instrum. Methods Phys. Res., Sect. B, Beam Interact. Mater. Atoms 267, 1460 (2009)

50. A. Rivera, M.L. Crespillo, J. Olivares, G. García, F. Agulló-López, Nucl. Instrum. Methods Phys. Res., Sect. B, Beam Interact. Mater. Atoms 268, 2249 (2010) 